\title{
A new perspective on GCRT J1745-3009
}

\author{
H. Spreeuw ${ }^{1}$, B. Scheers ${ }^{1}$, R. Braun ${ }^{2,6}$, R. A. M. J. Wijers ${ }^{1}$, J. C. A. Miller-Jones ${ }^{3}$, B. W. Stappers ${ }^{4,6}$, and R. P. Fender ${ }^{5}$ \\ 1 Astronomical Institute "Anton Pannekoek", University of Amsterdam, PO Box 94249, 1090 GE Amsterdam, The Netherlands \\ e-mail: [j.n.spreeuw;1.h.a.scheers;r.a.m.j.wijers] @uva.nl \\ 2 Australia Telescope National Facility, CSIRO, PO Box 76, Epping NSW 1710, Australia \\ e-mail: robert.braun@csiro.au \\ 3 Jansky Fellow, National Radio Astronomy Observatory, 520 Edgemont Road, Charlottesville, VA 22903, USA \\ e-mail: jmiller@nrao.edu \\ ${ }^{4}$ University of Manchester, Jodrell Bank Observatory, Macclesfield, Cheshire SK11 9DL, UK \\ e-mail: ben.stappers@manchester.ac.uk \\ 5 School of Physics and Astronomy, University of Southampton, Highfield, Southampton, SO17 1BJ, UK \\ e-mail: rpf@phys.soton.ac.uk \\ 6 ASTRON, PO Box 2, 7990 AA Dwingeloo, The Netherlands
}

Received 23 June 2008 / Accepted 12 April 2009

\section{ABSTRACT}

Context. Reports on a transient source about $1.25^{\circ}$ south of the Galactic Centre motivated these follow-up observations with the WSRT and the reinvestigation of archival VLA data. The source GCRT J1745-3009 was detected during a 2002 Galactic Centre monitoring programme with the VLA at $92 \mathrm{~cm}$ by five powerful 10-min bursts with a 77-min recurrence while apparently lacking any interburst emission.

Aims. The WSRT observations were performed and archival VLA data reduced to detect GCRT J1745-3009 again at different epochs and frequencies, to constrain its distance, and to determine its nature. We attempted to extract a more accurate lightcurve from the discovery dataset of GCRT J1745-3009 to rule out some of the models that have been suggested. We also investigated the transient behaviour of a nearby source.

Methods. The WSRT data were taken in the "maxi-short" configuration, using $10 \mathrm{~s}$ integrations, on 2005 March 24 at $92 \mathrm{~cm}$ and on 2005 May 14/15 at $21 \mathrm{~cm}$. Five of the six VLA observations we reduced are the oldest of this field in this band.

Results. GCRT J1745-3009 was not redetected. With the WSRT we reached an rms sensitivity of $0.21 \mathrm{mJy}^{\text {beam }}{ }^{-1}$ at $21 \mathrm{~cm}^{2}$ and $3.7 \mathrm{mJy}$ beam $^{-1}$ at $92 \mathrm{~cm}$. Reanalysis of the discovery observation data resulted in a more accurate and more complete lightcurve. The five bursts appear to have the same shape: a steep rise, a more gradual brightening, and a steep decay. We found variations in burst duration of order $\simeq 3 \%$. We improved the accuracy of the recurrence period of the bursts by an order of magnitude: $77.012 \pm$ $0.021 \mathrm{~min}$. We found no evidence of aperiodicity. We derived a very steep spectral index: $\alpha=-6.5 \pm 3.4$. We improved the $5 \sigma$ upper limits for interburst emission and fractional circular polarisation to $31 \mathrm{mJy}^{-1}$ beam $^{-1}$ and $8 \%$, respectively. Any transient behaviour of a nearby source could not be established.

Conclusions. Models that predict symmetric bursts can be ruled out, but rotating systems are favoured, because their periodicity is precise. Scattering constraints imply that GCRT J1745-3009 cannot be located far beyond the GC. If this source is an incoherent emitter and not moving at a relativistic velocity, it must be closer than $14 \mathrm{pc}$.

Key words. stars: individual: GCRT J1745-3009 - stars: binaries: close - stars: neutron - radio continuum: stars

\section{Introduction}

Reports of a peculiar radio transient, GCRT J1745-3009, about $1.25^{\circ}$ south of the Galactic Centre (Hyman et al. 2005, $2006,2007)$ and the suggestion that this may be the prototype of a new class of particularly bright, coherently emitting radio transients have led to speculation about its nature. In particular, the 77 min recurrence of the Jy level bursts was attributed to a period of rotation (Zhang \& Gil 2005), revolution (Turolla et al. 2005) and precession (Zhu \& Xu 2006). A nulling pulsar and an "X-ray quiet, radio-loud" X-ray binary have also been suggested (Kulkarni \& Phinney 2005), as well as an exoplanet and a flaring brown dwarf (Hyman et al. 2005). The discovery has led to follow-up observations and re-examination of archival data at both $92 \mathrm{~cm}$ and other bands. Those did not reveal a source (Zhu \& Xu 2006; Hyman et al. 2005, 2006), with two exceptions (Hyman et al. 2006, 2007). Both of the redetections were single bursts, possibly due to the sparse sampling of these observations. The first redetection was possibly the decaying part of a bright ( $0.5 \mathrm{Jy}$ level) burst that was detected at the first two minutes of a ten minute scan. The second redetection was a faint short ( $\simeq 2 \mathrm{~min}$ ) burst that was completely covered by the observation. The average flux density during the burst was only $57.9 \pm 6.6 \mathrm{mJy} / \mathrm{beam}$. This redetection also showed evidence for a very steep spectral index $(\alpha=-13.5 \pm 3.0)$.

The source has only been detected at three epochs, separated by less than 18 months, all at $92 \mathrm{~cm}$, while the source was not detected in this band at 33 epochs over a period of more than 16 years (see Hyman et al. 2006, Table 1) nor in any other band, ever. We observed the field containing GCRT J1745-3009 using eight 10-MHz IFs in the $92 \mathrm{~cm}$ band because its possible association with the supernova remnant G359.1-0.5 would mean that this source is about as far as the Galactic Center. That, in turn, implies a substantial dispersion measure (DM) that will become apparent as a delay of several seconds between the highest frequency IF and the lowest. This would be measurable if the bursts had some sufficiently sharp feature. An observation at $21 \mathrm{~cm}$ 
was performed to make use of the lower Galactic confusion and high sensitivity of the WSRT. We reanalysed five archival VLA datasets taken between 1986 and 1989 and the 2002 discovery dataset. All of these except the last were pointed at SgrA. Two of them, both obtained in A-configuration, had not been imaged before with the proper three-dimensional image restoration techniques. The complete set of observations we reduced is specified in Table 1.

\section{Data reduction}

\subsection{General}

We used AIPS (Greisen 2003) for the reduction of all datasets.

\subsection{The $92 \mathrm{~cm}$ WSRT observations on 2005 March 24}

The WSRT $92 \mathrm{~cm}$ observations on 2005 March 24 started at UT 01:22 with the observation of the calibration source 3C 295. We acquired data from the target field from 02:33 until 07:50 using $10 \mathrm{~s}$ integrations, with eight $10-\mathrm{MHz}$ IFs, consisting of 128 channels, each $78.125 \mathrm{kHz}$ wide, separated $8.75 \mathrm{MHz}$ from each other and centered on frequencies ranging from 315.4 to 376.6 MHz. RFI was excised from the spectral line data using the AIPS task "SPFLG", while remaining RFI was removed from the continuum data using the AIPS task "TVFLG".

Calibration was done in four steps. First we determined the variation in system temperature as a function of time (and therefore also position on the sky), using the intermittent firing of a stable noise source. Next we performed a bandpass calibration using the AIPS task "BPASS". We applied the bandpass solution using the AIPS task "SPLAT", producing a continuum file with one channel per IF. After that, we performed an external absolute gain calibration using an assumed flux of $61.5 \mathrm{Jy}$ for 3C 295 in the lowest frequency IF, by running the AIPS tasks "SETJY" and "CALIB". "SETJY" was set to use the absolute flux density calibration determined by Baars et al. (1977) and the latest (epoch 1999.2) polynomial coefficients for interpolating over frequency as determined at the VLA by NRAO staff. Finally, we self-calibrated the data for time variations in the relative complex gain phase and amplitude.

Theoretically, we should be able to reach a thermal noise level of $0.15 \mathrm{mJy} /$ beam in a $5 \mathrm{~h}$ integration, or at least the nominal beam confusion noise limit of $0.3 \mathrm{mJy} /$ beam. However, we did not attain this sensitivity due to the limited $u v$-coverage, RFI, and the existence of bright diffuse emission in the field. The latter compromises both self-calibration and image quality. This could be remedied to some extent by excluding spacings below a certain limit $\left(u v_{\min }>\right.$ some multiple of $\lambda$, the wavelength). We chose a $u v_{\min }$ of $1.0 k \lambda$ to eliminate the bulk of the diffuse emission, which could not be deconvolved with the available $u v$-coverage. SgrA and Tornado are the dominant sources in the field, their sidelobes contributed significantly to the image noise level of $9.0 \mathrm{mJy}^{\text {beam }}{ }^{-1}$ at the location of GCRT J1745-3009. These and other sources were deconvolved in an image with an asymmetrical cell size $\left(10^{\prime \prime} \times 60^{\prime \prime}\right)$. We chose to do so because a symmetrical cell size would yield a very elongated synthesized beam, this would hamper the deconvolution process. We subtracted the clean components of all sources from the $u v$-data before imaging the residual data with a symmetrical cell size. To lower the noise from the sidelobes of the two poorly subtracted extended sources, this final residual image was made by imposing a more severe lower limit of $2.5 k \lambda$ on the spacings, which resulted in a noise level of $3.7 \mathrm{mJy}$ beam $^{-1}$. That final image was made from only $7 \%$ of the recorded visibilities.

In retrospect, it is possible that the self-calibration process was adversely affected by bandwidth smearing, particularly because SgrA and Tornado were located far from the phase tracking center. Bandwidth smearing could have been diminished by keeping many channels per IF in "SPLAT". SgrA and Tornado were close to the half power beam width (HPBW). This also hampers self-calibration because the frequency dependence of the primary beam attenuation is much stronger near the HPBW than near the pointing center. It could have been fixed to some extent by running self-calibration per IF, at the expense of signal to noise. These flaws, the poor uv-coverage, the exclusion of many spacings and the Galactic plane contribution to the system temperature explains why the achieved noise level is still well above the thermal noise limit of $0.68 \mathrm{mJy} \mathrm{beam}^{-1}$ for this number of visibilities, imaging bandwidth and IFs (see Table 1), for a circular $60^{\prime \prime}$ beam towards cold sky.

\subsection{The $21 \mathrm{~cm}$ WSRT observations on 2005 May 14/15}

The 2005 May 14/15 observations at $21 \mathrm{~cm}$ started at UT 22:33 with the observation of the calibration source $3 \mathrm{C} 286$. We acquired data from the GCRT J1745-3009 field from 23:09 until 03:46 using $10 \mathrm{~s}$ integrations, with eight 20-MHz IFs, separated $17 \mathrm{MHz}$ from each other and centered on frequencies ranging from 1265 to $1384 \mathrm{MHz}$. The calibration was done in the same way as for the $92 \mathrm{~cm}$ WSRT observation. The assumed flux for the calibrator source 3C 286 in the lowest frequency IF was $15.6 \mathrm{Jy}$. Theoretically, the rms sensitivity of these observations could be as low as about $21 \mu \mathrm{Jy} \mathrm{beam}^{-1}$, for a $4.6 \mathrm{~h}$ integration. However, as for the $92 \mathrm{~cm}$ WSRT data, we excluded short spacings to eliminate most of the diffuse emission, which was necessary for successful self-calibration. The rms noise level in the final residual image was about $210 \mu \mathrm{Jy} \mathrm{beam}^{-1}$. That noise level is partly due to the loss of data: the exclusion of spacings below $2.5 \mathrm{k} \lambda$ and the excision of RFI. The total loss of visibilities up to the final image was as high as $55 \%$. With this number of visibilities and with the imaging bandwidth and IFs as mentioned in Table 1 , the theoretical thermal noise limit is $45 \mu \mathrm{Jy} \mathrm{beam}^{-1}$ for a circular 13" beam towards cold sky.

\subsection{The $92 \mathrm{~cm}$ VLA discovery dataset of 2002 September 30/October 1}

The specifics of the 2002 discovery dataset are shown in Table 1. We started its reduction with the flagging of 4 of the 27 antennas. Also, we flagged individual spectral channels per baseline, per IF and per polarisation product for all or part of the observing time, using the AIPS task "SPFLG". We flagged small portions, of $1 \mathrm{~min}$ or more, of data at the beginning and end of each scan using the AIPS task "QUACK". We also clipped data contaminated by RFI using the AIPS task "CLIPM". Next, we performed an external absolute gain calibration with an assumed flux of 25.9 Jy for 3C 286 in the lowest frequency IF. This flux was determined by running the AIPS task "SETJY", using the absolute flux density calibration determined by Baars et al. (1977) and the latest (epoch 1999.2) VLA polynomial coefficients for interpolating over frequency. We determined gain phase and gain amplitude solutions for both the primary calibrator 3C 286 and the phase calibrator 1711-251, using the AIPS task "CALIB". This task was run using all spacings for the primary calibrator and spacings longer than $1 k \lambda$ for the phase calibrator. The AIPS 
Table 1. Specifications of these observations.

\begin{tabular}{ccccccccccc}
\hline \hline No. & $\begin{array}{c}\text { Date } \\
\text { (yymmdd) }\end{array}$ & $\begin{array}{c}\text { Telescope } \\
\text { (+conf.) }\end{array}$ & $\begin{array}{c}\text { Number } \\
\text { of } \\
\text { antennas }^{1}\end{array}$ & $\begin{array}{c}\text { Number } \\
\text { of } \\
\text { IFs }\end{array}$ & $\begin{array}{c}\text { Number } \\
\text { of chann. } \\
\text { per IF }\end{array}$ & $\begin{array}{c}\text { Bandwidth } \\
\text { per IF } \\
\text { (MHz) }\end{array}$ & $\begin{array}{c}\text { Number of } \\
\text { pol.prod. } \\
\text { per IF }\end{array}$ & $\begin{array}{c}\text { Ch.width } \\
\text { for ima- } \\
\text { ging (kHz) }\end{array}$ & $\begin{array}{c}\text { Tot.BW } \\
\text { for ima- } \\
\text { ging (MHz) }\end{array}$ & $\begin{array}{c}\text { On-source } \\
\text { time (h) }\end{array}$ \\
\hline 1 & 860329 & VLA A & 11 & 1 & 127 & 3.1 & 1 & 98 & 2.5 & 4.6 \\
2 & 860805 & VLA B & 8 & 1 & 127 & 0.8 & 1 & 98 & 0.7 & 4.9 \\
3 & 861226 & VLA C & 15 & 1 & 63 & 0.8 & 1 & 98 & 0.7 & 6.2 \\
4 & 881203 & VLA A & 22 & 2 & 7 & 1.4 & 1 & 195 & 2.7 & 5.7 \\
5 & 890318 & VLA B & 27 & 2 & 7 & 0.7 & 1 & 98 & 1.4 & 5.3 \\
6 & 020930 & VLA CnB & 22 & 2 & 31 & 3.0 & 2 & 98 & 8.2 & 5.3 \\
7 & 050324 & WSRT & 12 & 7 & 128 & 10.0 & 4 & 6328 & 89 & 5.3 \\
8 & 050514 & WSRT (21cm) & 14 & 6 & 64 & 20.0 & 4 & 12813 & 154 & 4.6 \\
\hline
\end{tabular}

${ }^{1}$ This is the nummer of antennas after flagging; ${ }^{2}$ this is the number of IFs after flagging averaged over the RR and LL polarisation products, if both are available; ${ }^{3}$ this is total bandwidth for Stokes I imaging, we added RR and LL bandwidth.

task "GETJY" determines the flux of the secondary calibrator from those gain solutions and the flux of the primary calibrator. "GETJY" found a flux of 11.1 Jy for 1711-251 at the highest frequency IF (327.5 MHz). The gain solutions were interpolated using the AIPS task "CLCAL".

Next, we used 3C 286 to find a bandpass solution. In doing so, we applied the interpolated gain solutions from "CLCAL" for spacings longer than 500 wavelengths $\left(u v_{\min }>0.5 k \lambda\right)$. For one of the antennas no visibilities were recorded during the scan of $3 \mathrm{C} 286$. Hence, no bandpass solution could be found for this antenna and only 22 antennas were left for imaging. We applied the gain and bandpass solution to 20 of the total of 31 available channels using the AIPS task "SPLAT". Every two channels were averaged.

Next, we performed 18 iterations of phase only selfcalibration, using initial solution intervals of $5 \mathrm{~min}$, gradually decreasing down to $1 \mathrm{~min}$. We used $195 \mathrm{kHz}$ channels for imaging and a cellsize of $4^{\prime \prime}$. We used $85512 \times 512$ pixel facets to cover the primary beam and no facets for outlier fields. We performed an amplitude and phase self-calibration and we produced the final model from the spectral averaged dataset. After that, we reran "SPLAT" on the line data, but this time without spectral averaging, selecting $21 \times 97 \mathrm{kHz}$ of the available channels. We phase self-calibrated the new dataset using the acquired model from the spectral averaged data. Next, we imaged and deconvolved our phase self-calibrated dataset using 61 facets to cover the primary beam and 22 facets for the outlier fields. This time we used $256 \times 256$ pixel facets with a pixel size of $10^{\prime \prime}$. We self-calibrated again, but this time we solved for amplitude and phase, using a solution interval of $1 \mathrm{~min}$. The total average gain was normalized in this process. We imaged and deconvolved $450 \mathrm{Jy}$ of total flux from the amplitude and phase self-calibrated dataset to make our final model. Figure 1 shows the central facet of this model after correction for primary beam attenuation. We noticed that SgrA is by far the brightest source in the field and that it is near the half power beam point. We anticipated that the calibration of the $u v$ data could be optimized by applying separate gain solutions to the clean components of the facet with SgrA, so we ran the AIPS runfile PEELR on the clean components of the facet of SgrA, solving for gain amplitudes and phases on a timescale of $10 \mathrm{~s}$. We subtracted the clean components from the peeled data using the AIPS task "UVSUB" and we determined the position of GCRT J1745-3009 in our final model using the AIPS task "IMFIT". We shifted the phase stopping centre to this position using the AIPS task "UVFIX" and we averaged all spectral channels using the AIPS task "SPLIT". We did a final edit using the AIPS task "CLIP" and set $u v_{\min }=1.0 \mathrm{k} \lambda$. We ran the AIPS task "DFTPL" on this final residual dataset to produce our lightcurves. We did not correct the output of "DFTPL" for primary beam attenuation because GCRT J1745-3009 was about 13' from the pointing center. Primary beam attenuation for this angular separation is only $1.8 \%$.

In retrospect, it turned out that both the amplitude and phase (A\&P) self-calibration and the peeling of SgrA had negligible effect on the burst shapes in the final lightcurves. So the dataset could be reduced in a standard way, except perhaps for the large number of selfcal iterations and the exclusion of a rather large number of antennas, 5 of the 27 antennas being excluded for the entire observation.

\section{The source on the opposite side of the supernova remnant}

The source northeast of the supernova remnant G359.1-0.5, indicated by a box in Fig. 1 is resolved in VLA A configuration. From a combination of three VLA datasets, two in A configuration and one in $\mathrm{B}$ configuration, this source was detected with a peak flux density of $17.1 \pm 2 \mathrm{mJy}^{\mathrm{beam}}{ }^{-1}$ and an integrated flux of 47.6 mJy (see Nord et al. 2004, Table 2, source 72). Apparently the synthesized beam of the combination of these datasets $\left(12^{\prime \prime} \times 7^{\prime \prime}\right)$ resolves this source. As noted in the caption of Fig. 1, the peak flux density we derived from the 2002 discovery observation is $91 \pm 14 \mathrm{mJy}^{\text {beam }}{ }^{-1}$. A large fraction of the difference with the integrated flux measurement by Nord et al. (2004) is probably caused by extended emission. Indeed, when we exclude the shortest spacings, $u v_{\min }=1.0 \mathrm{k} \lambda$, we find a much lower peak flux density of $73 \pm 5 \mathrm{mJy}^{\text {beam }}{ }^{-1}$. The remaining difference may also come from extended emission that is picked up differently by these observations.

However, the main reason that this source drew our attention is its absence in a high dynamic range image of the Galactic Centre at $92 \mathrm{~cm}$ with a noise level of about $5 \mathrm{mJy} \mathrm{beam}^{-1}$ and an angular resolution of 43" (see LaRosa et al. 2000, Fig. 11, hereafter called the LaRosa map). The datasets used for the LaRosa map were taken on 1986 August 5 (B conf., 8 antennas) and 1986 December 26 (C conf., 15 antennas), 1987 March 25 (D conf., 15 antennas) and 1989 March 18 (B conf., 27 antennas). Our reduction of the 1989 March 18 data shows the source at the $\geq 6 \sigma$ level, a Gaussian fit gave a peak flux density of $53 \pm 7 \mathrm{mJy}$ beam $^{-1}$. Here, the size of the synthesized beam is $27^{\prime \prime} \times 14^{\prime \prime}$ while we set $u v_{\min }$ to $2.0 \mathrm{k} \lambda$. This clear detection indicates that the non-detection of the source in the LaRosa map is 
Table 2. Flux measurements at $92 \mathrm{~cm}$ (unless otherwise noted) for detections and nondetections of GCRT J1745-3009 at $\alpha=17 \mathrm{~h} 45 \mathrm{~m} 5.15 \mathrm{~s}$, $\delta=-30^{\circ} 09^{\prime} 52.7^{\prime \prime}$ (Kaplan et al. 2008). Corrections for primary beam attenuation and bandwidth smearing have been applied where appropiate.

\begin{tabular}{ccccccc}
\hline \hline No. & $\begin{array}{c}\text { Date } \\
\text { (yymmdd) }\end{array}$ & $\begin{array}{c}\text { Telescope } \\
\text { (+conf. })\end{array}$ & $\begin{array}{c}\text { Peak flux density } \\
(\mathrm{mJy} / \text { beam })\end{array}$ & $\begin{array}{c}\text { Error on fit } \\
(\mathrm{mJy} / \text { beam })\end{array}$ & $\begin{array}{c}\text { rms noise } \\
(\mathrm{mJy} / \text { beam })\end{array}$ & $\begin{array}{c}\text { Resolution } \\
\left({ }^{\prime \prime} \times{ }^{\prime \prime}\right)\end{array}$ \\
\hline 1 & 860329 & VLA A & -49 & 27 & 18 & $10 \times 4$ \\
2 & 860805 & VLA B & -19 & 20 & $100^{1}$ & $44 \times 33$ \\
3 & 861226 & VLA C & -26 & 29 & $100^{1}$ & $105 \times 54$ \\
4 & 881203 & VLA A & -18 & 15 & 15 & $12 \times 6$ \\
5 & 890318 & VLA B & 41 & 19 & 8 & $27 \times 14$ \\
6 & 020930 & VLA CnB & $110^{2}$ & $8^{2}$ & $8^{3}$ & $44 \times 36$ \\
7 & 050324 & WSRT & 5 & 4 & 4 & $148 \times 27$ \\
8 & 050514 & WSRT $(21 \mathrm{~cm})$ & -0.3 & 0.2 & 0.2 & $68 \times 9$ \\
\hline
\end{tabular}

${ }^{1}$ The formal rms noise levels in these two maps are $19 \mathrm{mJy} \mathrm{beam}^{-1}$ and $69 \mathrm{mJy}_{\text {beam }}{ }^{-1}$ for the 1986 August 5 and December 26 observations respectively, (much) lower than the indicated value of $100 \mathrm{mJy} \mathrm{beam}^{-1}$. However, many bright compact sources that should be detectable in these maps, are not due to the very poor uv coverage of this observation. We accounted for this by replacing the rms noise by a higher number, in this way giving a very crude representation of these missing sources.

${ }^{2}$ Here we did not tie the clean beam fit to the position from Kaplan et al. (2008), but set the AIPS task "IMFIT" to solve for peak flux density as well as position in the residual image.

${ }^{3}$ This is the average noise in the residual image.

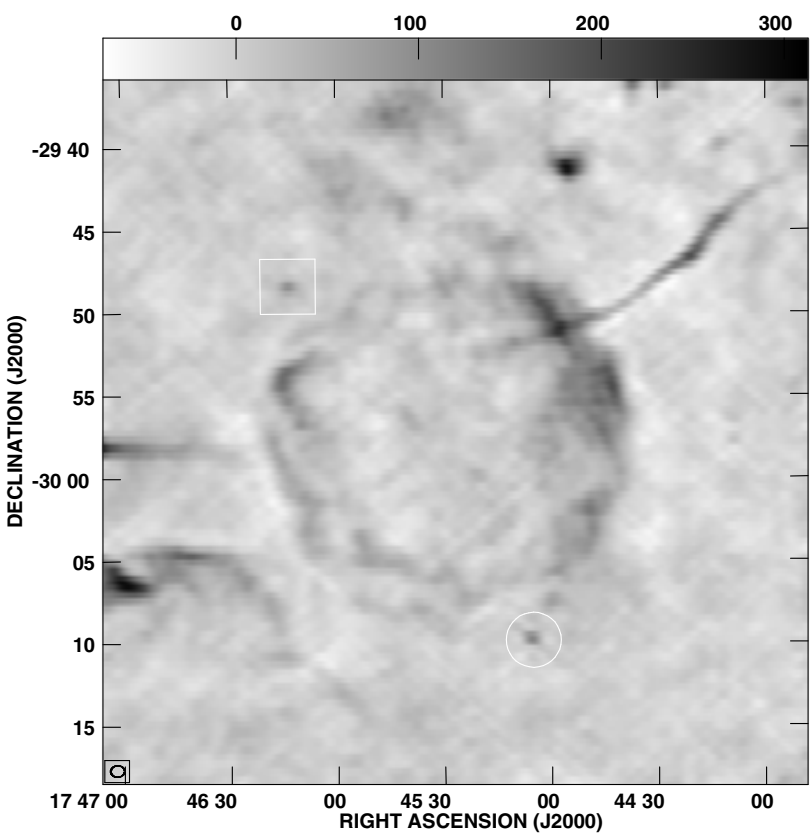

Fig. 1. The supernova remnant G359.1-0.5 with "The Snake" to the northwest, from our reduction of the GCRT J1745-3009 discovery observation on 2002 September 30/October 1 with the VLA in CnB configuration. This observation revealed this transient, indicated by a circle, for the first time (see Hyman et al. 2005). Noise levels in this image vary from 5 to $13 \mathrm{mJy}$ beam ${ }^{-1}$ across the image. A Gaussian fit to the unresolved GCRT J1745-3009 gives a peak flux density of only $116 \pm$ $14 \mathrm{mJy}$ beam $^{-1}$ because the five Jy-level bursts have been averaged over about $6 \mathrm{~h}$ of observation. A Gaussian fit to the source to the northeast of the supernova remnant, indicated by the box, gives a peak flux density of $91 \pm 14 \mathrm{mJy}_{\text {beam }}^{-1}$. Correction for primary beam attenuation has been applied.

probably not due to transience. More likely, the source is concealed in the LaRosa map by a negative background peak.

\section{Overview of flux measurements of GCRT J1745-3009}

We hoped to redetect GCRT J1745-3009 with the WSRT, with some of the VLA observations mentioned in the previous section and with two additional A configuration observations from the VLA archive. We did not redetect the source, but we measured its flux at its position in all of the seven maps. Specifics of these observations are shown in Table 1. Note that the onsource time for the two WSRT observations is comparable to the VLA observations, despite the limited time for which the WSRT can observe this low declination source. The reason for this is that the WSRT in general does not need to observe secondary calibrators. The results of the flux measurements at these epochs and at the time of the discovery are shown in Table 2. For the seven nondetections, we fitted the restoring beam to the position reported by Kaplan et al. (2008). We have also imaged $10 \mathrm{~min}$ subsets of the residual data from the five 1986-1989 observations to look for isolated bursts, but we found none.

We merged our results from Table 2 with those from a recent overview of observations since 1989 (see Hyman et al. 2006, Table 1) together with the results from the second redetection (Hyman et al. 2007) to produce a plot of $5 \sigma$ flux upper limits on quiescent emission from GCRT J1745-3009 in the $92 \mathrm{~cm}$ band (see Fig. 2). In order to derive appropriate values, we scaled the 10 -min scan sensitivities mentioned (20 and $10 \mathrm{mJy}^{\text {beam }}{ }^{-1}$ for the VLA and the GMRT respectively, after correction for primary beam attenuation) with the square root of the observing bandwidth, taking $6.2 \mathrm{MHz}$ as the base. The sensitivities for complete observations were also scaled with the square root of the total on-source time. We note that the 1989 March 18 observation was already analysed by Hyman et al. (2006) and their reduction led to slightly more constraining values, so we adopted these in Fig. 2. Here, we took account of the fact that the total bandwidth of that observation was actually $1.4 \mathrm{MHz}$ instead of the $12.5 \mathrm{MHz}$ mentioned in their Table 1. Consequently, we derived $5 \sigma$ upper limits of $5 \cdot 20 \cdot \sqrt{6.2 / 1.4}=210$ mJy beam $^{-1}$ and $5 \cdot 20 \cdot \sqrt{6.2 / 1.4} / \sqrt{5.3 \cdot 6}=37 \mathrm{mJy}^{\text {beam }}{ }^{-1}$, for those $10-\mathrm{min}$ scans and for that complete observation, respectively.

The lowest noise level of all $92 \mathrm{~cm}$ observations, about $6 \mathrm{mJy}$ beam $^{-1}$ in a 2 min interval, was achieved at the time of the second redetection, with the GMRT on 2004 March 20 (Hyman et al. 2007). This is actually the only observation that could have detected bursts of this kind and only by making 2 min scan averages. None of the observations included in Fig. 2 can detect the 2004 burst (Hyman et al. 2007) in 10 minute averages at the $5 \sigma$ noise level. 


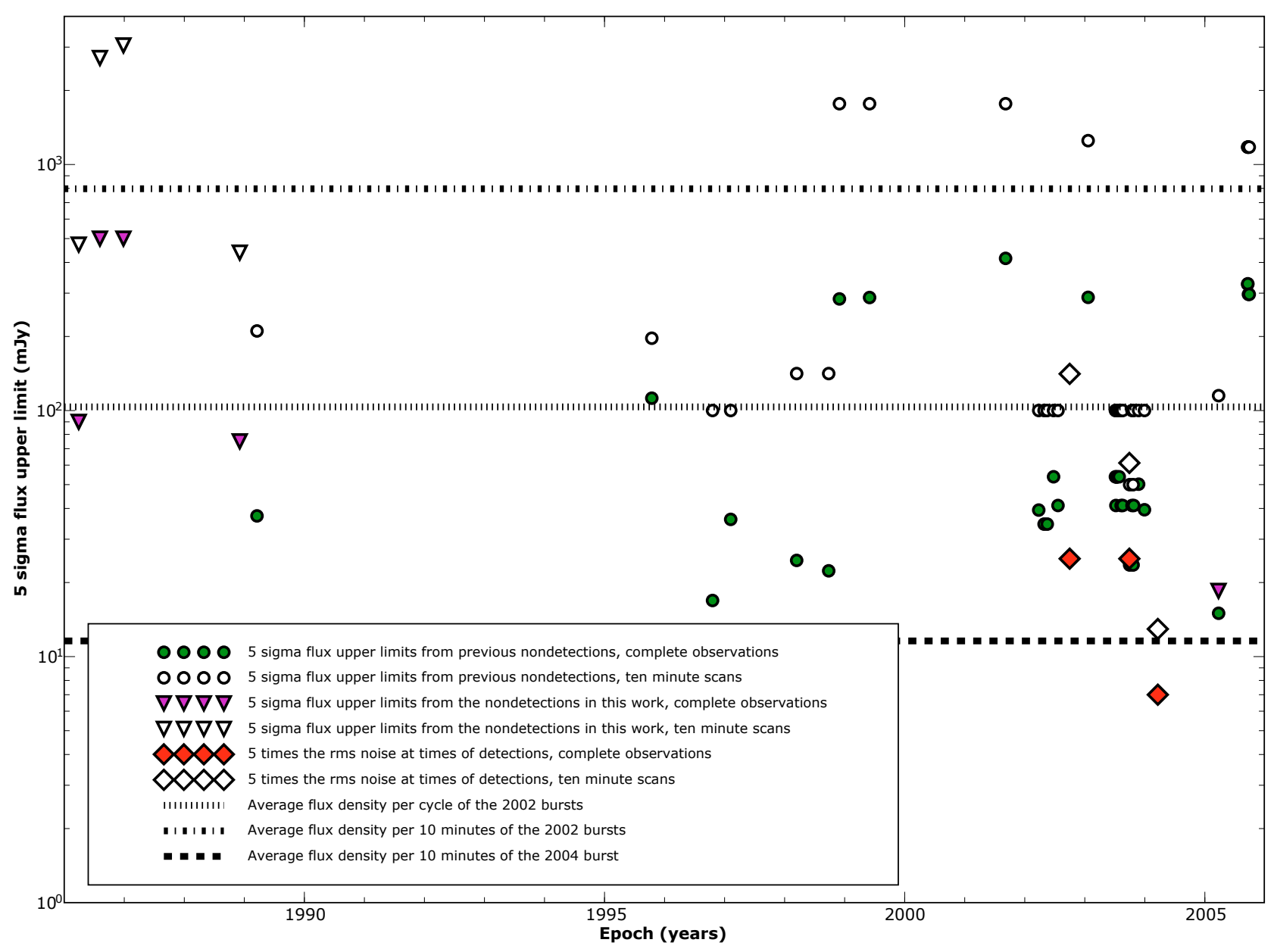

Fig. 2. Approximate detection thresholds (5 $\sigma$ noise levels) at the location of GCRT J1745-3009 of 41 Galactic Center observations at $92 \mathrm{~cm}$ over two decades. For the WSRT observation at $92 \mathrm{~cm}$, the $10 \mathrm{~min}$ scan sensitivity is not indicated, since the snapshot point spread function (psf) of a linear array does not allow to do this accurately. The observations in this plot start on 1986 March 29 and end on 2005 September 27.

The WSRT 2005 May 14/15 $5 \sigma$ upper limit at $21 \mathrm{~cm}$ $\left(1.05 \mathrm{mJy}\right.$ beam $\left.^{-1}\right)$ was less constraining than the VLA upper limit at that wavelength on 2005 March 25 (0.4 mJy beam ${ }^{-1}$, see Hyman et al. 2006). $21 \mathrm{~cm}$ observations are not included in Fig. 2.

\section{Reanalysis of the $\mathbf{2 0 0 2}$ discovery dataset}

\subsection{Lightcurve}

The lightcurve that we extracted from the discovery dataset of GCRT J1745-3009 at the position derived in paragraph 5.3 is shown in Fig. 3. The bursts seem to have similar shapes: a steep rise, a gradual brightening and a steep decay, more consistent than the bursts shown in Fig. 1 of the discovery paper (Hyman et al. 2005). This lightcurve is twice as accurate as the original one. We also ran the AIPS task "DFTPL" with 5 s sampling, this is the integration time for the recording of the visibilities in the discovery dataset. We found no compelling evidence for interburst emission, not even on the shortest $(5 \mathrm{~s})$ timescale. We determined the recurrence interval between bursts by measuring the times of steepest rise for four of the bursts. Consecutive 1 min chunks of data were selected by a sliding window. For each chunk of data we determined its average slope by weighted linear regression. The weights come from the reciprocal of the noise variances from "DFTPL". The time corresponding to the steepest positive slope was then calculated as the weighted average of the timestamps in the datachunk. For the first burst, this method is illustrated in Fig. 4. Weighted linear regression also calculates the error bars of the times of steepest rise from the error bars of the data points. The times of steepest rise and the corresponding error bars are shown in Table 3 . The times mentioned in that table are relative to $20 \mathrm{~h} 50 \mathrm{~m} 00 \mathrm{~s}$ on 2002 September 30 (IAT). We then again applied the formulae for weighted linear regression to find the period between bursts and its $1 \sigma$ error. We found a period of 77.012 $\pm 0.021 \mathrm{~min}$ from the values in Table 3 . We have improved the error on the period by an order of magnitude (Hyman et al. 2006, paragraph 3 and caption of Fig. 3), but the period itself agrees with the previously determined period of $77.1 \mathrm{~m} \pm 15 \mathrm{~s}$. However, it is important to note that our method differs from the one used by Hyman et al. (2005). We have made no assumption with regard to the burst shapes in determining the period.

The residuals with respect to that fit are $0.097,-0.114,0.053$ and -0.007 min for the first, second, fourth and fifth burst, respectively. The residual for the second burst is the largest, $6.8 \mathrm{~s}$ "too late" with respect to the fit, this corresponds to $1.9 \sigma$, $\sigma=0.060 \mathrm{~min}$, this is the error on the time of steepest rise of the second burst.

We were also able to measure the times of steepest decay for four of the bursts in a similar manner, see Table 4. For three bursts we could measure both the time of steepest decay and the 


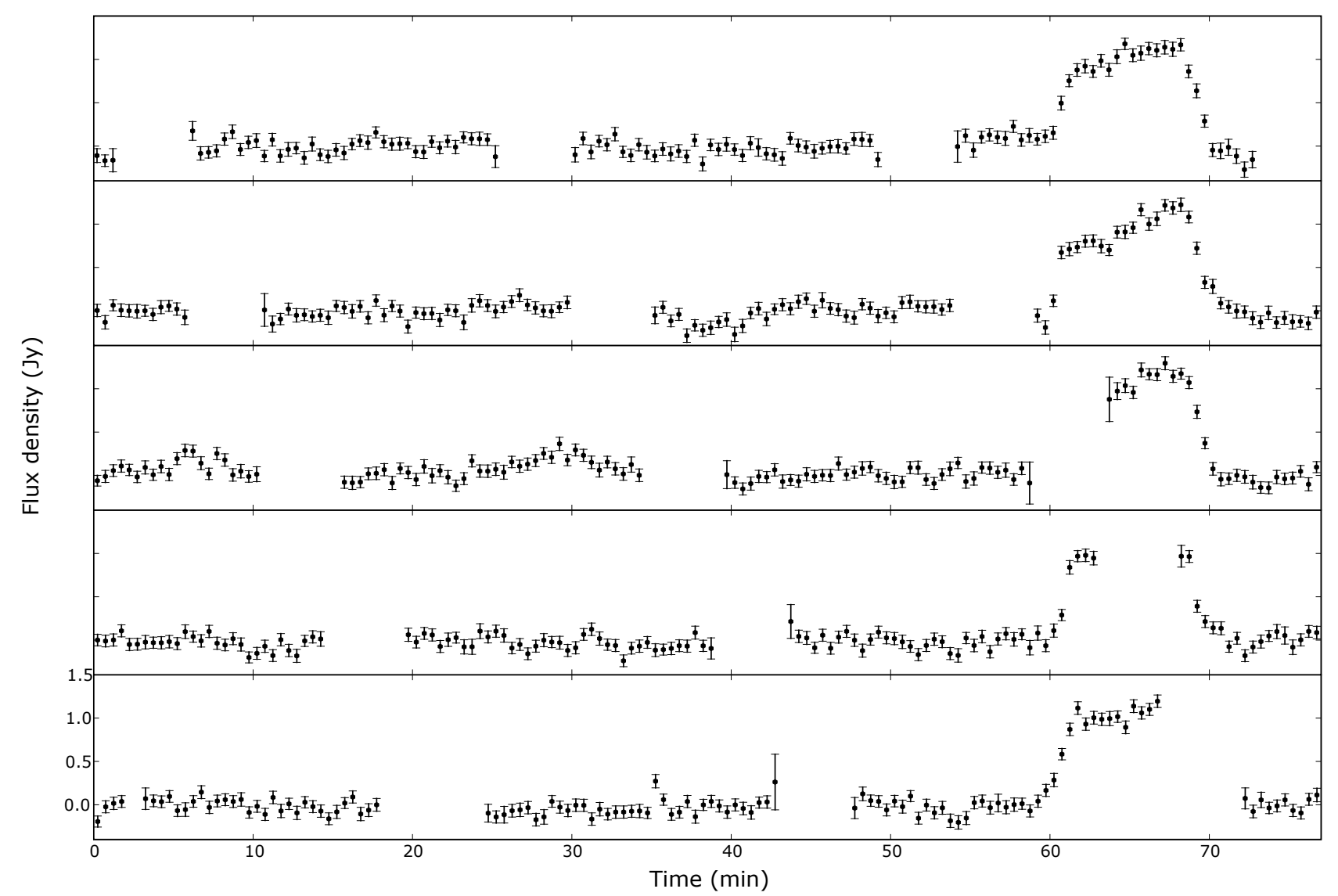

Fig. 3. The plot above shows the lightcurve from the discovery dataset of GCRT J1745-3009 with $30 \mathrm{~s}$ sampling. This plot is setup in the same way as the lightcurve in the discovery paper except for the flux density measurements between bursts. For those nondetections Hyman et al. (2005) showed $3 \sigma$ upper limits on interburst emission, we show the actual background flux density measurements. Also, we have folded the lightcurve at intervals of $77.012 \mathrm{~min}$ instead of $77.130 \mathrm{~min}$. The first interval is shown in the bottom panel, starting at 20h50m00s on $2002 \mathrm{September} 30$ (IAT). The average of all the error bars shown is $74 \mathrm{mJy}$. The gaps are due to phase calibrator observations.

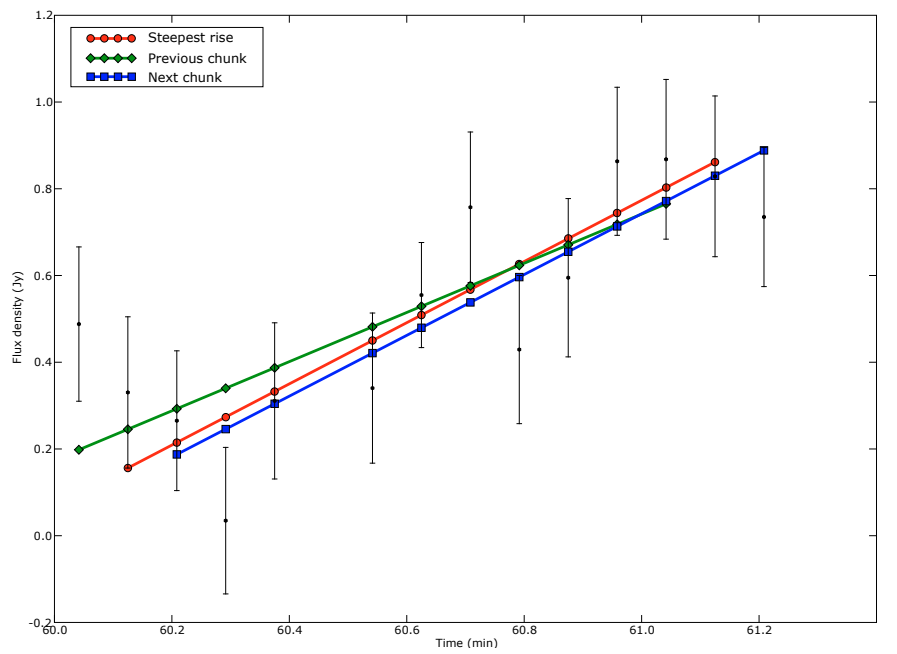

Fig. 4. This plot illustrates how the times of steepest rise for four of the bursts are determined. Weighted linear regression is performed on successive one minute chunks of data. The chunks have a maximum of $55 \mathrm{~s}$ of overlap time. Here the rising part of the first burst is shown.

time of steepest rise. In this way we found that the time between steepest rise and steepest decay varies. We found intervals of $8.29 \pm 0.08,8.87 \pm 0.09$ and $8.66 \pm 0.09 \mathrm{~min}$ for the second, fourth and fifth burst, respectively. So for the second burst the
Table 3. Measurements of times of steepest rise for four bursts.

\begin{tabular}{ccccc}
\hline \hline $\begin{array}{c}\text { Burst } \\
\text { number }\end{array}$ & $\begin{array}{c}\text { Time of steepest } \\
\text { rise }(\mathrm{min})\end{array}$ & $\begin{array}{c}1 \sigma \text { error } \\
(\mathrm{min})\end{array}$ & $\begin{array}{c}\text { Slope } \\
(\mathrm{Jy} / \mathrm{min})\end{array}$ & $\begin{array}{c}1 \sigma \text { error } \\
(\mathrm{Jy} / \mathrm{min})\end{array}$ \\
\hline 1 & 60.624 & 0.068 & 0.706 & 0.158 \\
2 & 137.848 & 0.060 & 0.828 & 0.175 \\
4 & 291.704 & 0.065 & 0.724 & 0.138 \\
5 & 368.776 & 0.065 & 0.743 & 0.150 \\
\hline
\end{tabular}

Table 4. Measurements of times of steepest decay for four bursts.

\begin{tabular}{ccccc}
\hline \hline $\begin{array}{c}\text { Burst } \\
\text { number }\end{array}$ & $\begin{array}{c}\text { Time of steepest } \\
\text { decay }(\mathrm{min})\end{array}$ & $\begin{array}{c}1 \sigma \text { error } \\
(\mathrm{min})\end{array}$ & $\begin{array}{c}\text { Slope } \\
(\mathrm{Jy} / \mathrm{min})\end{array}$ & $\begin{array}{c}1 \sigma \text { error } \\
(\mathrm{Jy} / \mathrm{min})\end{array}$ \\
\hline 2 & 146.136 & 0.041 & -1.125 & 0.146 \\
3 & 223.489 & 0.057 & -0.811 & 0.150 \\
4 & 300.578 & 0.067 & -0.717 & 0.150 \\
5 & 377.439 & 0.066 & -0.734 & 0.148 \\
\hline
\end{tabular}

interval between steepest rise and steepest decay is $3.45 \%$ less than the weighted mean of those three intervals. The significance of this deviation is $3.0 \sigma$.

We can use the derived period to fold the bursts in one plot, see Fig. 5. This plot shows that the bursts indeed have similar shapes. 


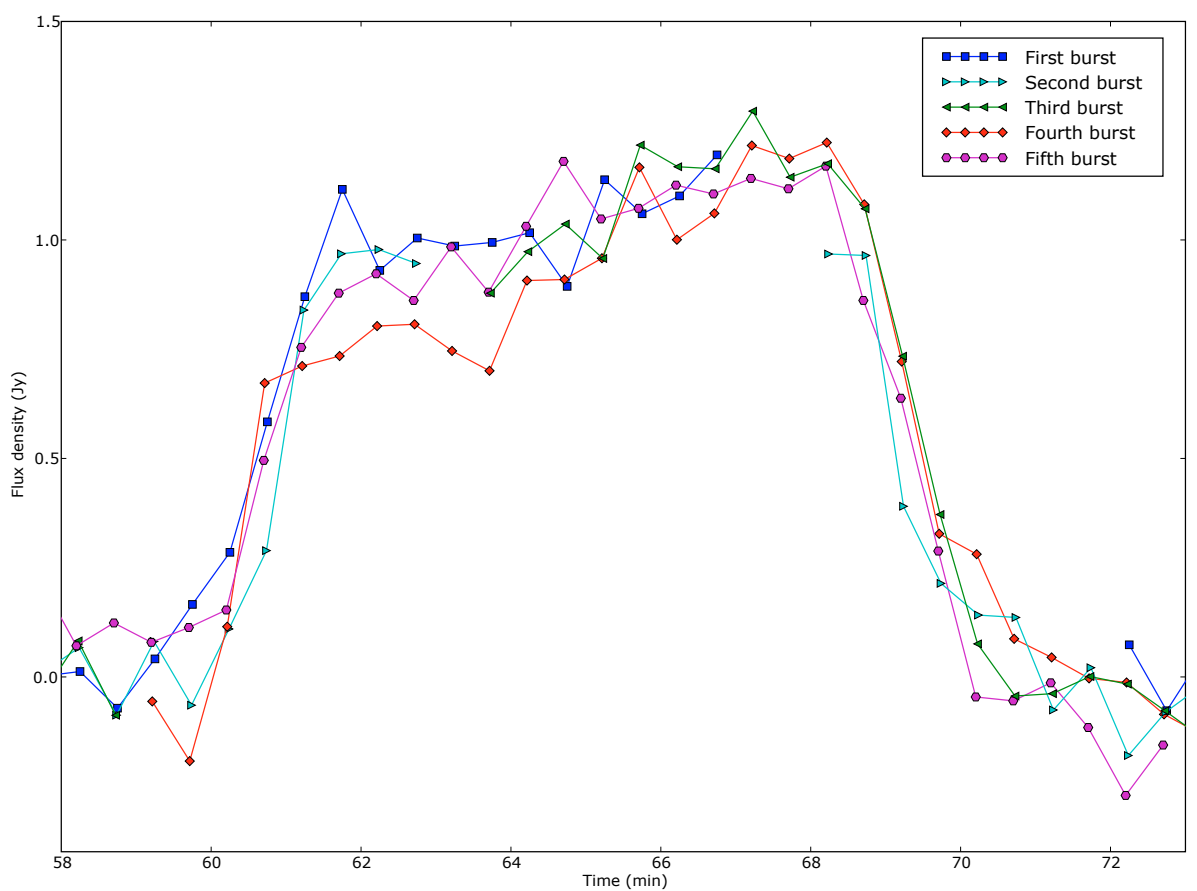

Fig. 5. The plot above shows the five bursts from the discovery dataset of GCRT J17453009 with $30 \mathrm{~s}$ sampling folded at intervals of $77.012 \mathrm{~min}$. Time is relative to $20 \mathrm{~h} 50 \mathrm{~m} 00 \mathrm{~s}$ on 2002 September 30 (IAT) (plus multiples of $77.012 \mathrm{~min}$ ).

\subsection{Implications for other observations}

Now that we have determined the periodicity of the bursts more accurately, we can check if other short GC observations at $92 \mathrm{~cm}$ before and after the discovery observation should have detected GCRT J1745-3009. The observation closest in time was taken on 2002 July 21 (see Hyman et al. 2006, Table 1). This was a $59.2 \mathrm{~min}$ scan starting $1719.75 \mathrm{~h}$ before the start of the bright part of the first burst in the discovery dataset. This corresponds to 1339.86 periods of $77.012 \mathrm{~min}$. Consequently, the source should not have been seen during that short scan and this was indeed the case (Hyman et al. 2006). However, there is a large uncertainty in calculating burst times over an interval as large as 71 days. The error is $0.021 \mathrm{~min} \cdot 1339=28 \mathrm{~min}$. From that uncertainty and Gaussian statistics, we calculated that the chance of having observed at least 5 min of bursting activity on 2002 July 21 was $74 \%$, assuming that GCRT J1745-3009 were bursting as during the discovery observation. If GCRT J1745-3009 was indeed active on 2002 July 21 , we can infer from the nondetection on that occasion that $P$, the recurrence interval between bursts is tightly constrained: $77.007 \mathrm{~min}<P<77.021 \mathrm{~min}$.

The next observation closest in time was taken on 2002 June 24. Its duration was only $34.5 \mathrm{~min}$, starting 1842.17 periods of $77.012 \mathrm{~min}$ before the start of the bright part of the first burst in the discovery dataset. During this observation we should have seen at least 6 min of a burst if we take into account the constraints on the period from the nondetection on 2002 July 21 . From the fact that we did not detect emission on 2002 June 24 we may conclude that activity started after this 34.5 min scan.

The first suitably pointed $92 \mathrm{~cm}$ observation after the discovery observation was taken on 2003 January 20 . The source was not detected, but the data were taken with the VLA in CD configuration. This implies that rms noise levels from 10-min scans are about 250 mJy beam $^{-1}$ (see Hyman et al. 2006, and Fig. 2 in this paper). Thus it is likely that GCRT J1745-3009 could not have been detected at the $5 \sigma$ level on 2003 January 20 , even if an individual ten minute scan were spaced in time such that it completely covered a burst. It may be that the activity continued until the summer of 2003 when three 59 min and four $34 \mathrm{~min}$ GC observations were performed with the VLA in A configuration. At least two of these scans are spaced in time such that if one covered the interval between two bursts, the other must have covered a complete burst. So we are sure that the recurrent bursting activity of GCRT J1745-3009 stopped before it was redetected on 2003 September 28.

In summary, the bursting activity with a period of $77.012 \mathrm{~min}$ as seen during the discovery observation must have started after 2002 June 24 and may have continued until the summer of 2003. Unfortunately, we cannot constrain the timespan of a recurrently bursting GCRT J1745-3009 to less than a year.

\subsection{Position and flux measurements; spectral index determination}

The most accurate position measurement, corresponding to the highest signal to noise ratio, can be achieved by selecting just the time intervals that cover the bursts. We found a peak flux density of $900 \pm 23 \mathrm{mJy}^{\mathrm{beam}}{ }^{-1}$ and this J2000 position: $\alpha=17 \mathrm{~h} 45 \mathrm{~m} 05.015 \mathrm{~s} \pm 0.045 \mathrm{~s}, \delta=-30^{\circ} 09^{\prime} 52.19^{\prime \prime} \pm 0.52^{\prime \prime}$. This position of GCRT J1745-3009 has not yet been corrected for ionospheric-induced refraction (see Nord et al. 2004, for some background). That correction, which is basically, but not exactly, a global position shift of all sources in the field, will significantly increase the uncertainty in the position of GCRT J1745-3009. Here, we just mention that in our maps the bright source SGR E46 is $0.33 \mathrm{~s}$ west and $0.89^{\prime \prime}$ north of the NVSS (Condon et al. 1998) position. The NVSS catalogue mentions a positional accuracy of $0.45^{\prime \prime}$ in right ascension and $0.6^{\prime \prime}$ in declination for this source. We consider the actual uncertainty for the given position of GCRT J1745-3009 to be 5" in both right ascension and declination.

Rms noise values in the map that constitutes our final model range between 5 and $13 \mathrm{mJy}_{\text {beam }}^{-1}$. We also made a map from the same data, but without short spacings $\left(u v_{\min }=1.0 \mathrm{k} \lambda\right)$. 
Table 5. Measurement of spectral index for each burst.

\begin{tabular}{ccc}
\hline \hline $\begin{array}{c}\text { Burst } \\
\text { number }\end{array}$ & $\begin{array}{c}\alpha \\
\left(S_{v} \propto v^{\alpha}\right)\end{array}$ & $1 \sigma$ error \\
\hline 1 & -9.9 & 6.7 \\
2 & -9.0 & 9.3 \\
3 & 0.9 & 8.7 \\
4 & -0.4 & 6.9 \\
5 & -12.3 & 6.9 \\
\hline
\end{tabular}

Noise levels then drop significantly, varying between 4 and $6 \mathrm{mJy}$ beam $^{-1}$ across the image. We removed the bursts and we made a cleaned image with the same spacings. The noise levels are somewhat higher now: between 5 and $7 \mathrm{mJy} \mathrm{beam}^{-1}$.

In order to derive an upper limit on interburst emission we fitted the clean beam to the position measured above. We found a peak flux density of $-0.6 \pm 6.4 \mathrm{mJy} \mathrm{beam}^{-1}$, after correction for primary beam attenuation $(1.8 \%)$. This gives a $5 \sigma$ upper limit on interburst emission of $31 \mathrm{mJy}^{\text {beam }}{ }^{-1}$. This is more than twice as constraining as the original upper limit.

Neglecting primary beam attenuation, we found a weighted mean flux of $103.5 \pm 2.9 \mathrm{mJy}^{\text {beam }}{ }^{-1}$ from the output of the AIPS task "DFTPL" on the residual data with full (5 s) sampling. We also ran "DFTPL" on this data for each of the five bursts and for each of the two IFs separately. We only selected times for which both IFs had fluxes and then calculated the natural logarithm of the ratio of the fluxes for each timestamp and the variance of that quantity. We then calculated the weighted mean of these logarithms for each burst. The spectral index and error bar for each burst are shown in Table 5, using the average frequencies of IF1 (327.5000 MHz) and IF2 (321.5625 MHz). The spectral indices and error bars of the individual bursts do not seem inconsistent with Gaussian statistics, so we calculated the weighted mean spectral index as well: $\alpha=-6.5 \pm 3.4$. This is not incompatible with the spectral indices found by Hyman et al. (2006, 2007, $\alpha=-4 \pm 5$ and $\alpha=-13.5 \pm 3.0)$, given the large error bars. The weighted mean of these three measurements is $\alpha=-9.4 \pm 2.1$.

\subsection{Circular polarisation}

We compared the lightcurves for left ("LL") and right ("RR") circular polarisation with $30 \mathrm{~s}$ sampling. Although there are occasional "LL" and "RR" flux differences during the bursts larger than the sums of the respective error bars, this is also seen in between the bursts. There is no compelling evidence for circularly polarised emission during any particular phase of the burst cycle. On the other hand, we cannot exclude it completely, because we have insufficient signal to noise in Stokes V.

From the residual data, we selected the times corresponding to the bursts and we made a Stokes V dirty image. We corrected for primary beam attenuation and fitted the clean beam to the position of GCRT J1745-3009 as we did in the previous paragraph to determine the upper limit for interburst emission. We measured a Stokes V of $-20 \pm 10 \mathrm{mJy}^{\text {beam }}{ }^{-1}$. Using the total intensity averaged over the bursts, $900 \pm 23 \mathrm{mJy} \mathrm{beam}^{-1}$, we found that the $5 \sigma$ upper limit on the fractional circular polarisation, $|V| / I$, is $8 \%$. Hyman et al. (2005) derived a weaker constraint of $15 \%$ on the fractional circular polarisation averaged over the bursts.

Despite the lack of evidence for circularly polarised emission in the discovery observation, it has been detected in the data from the 2003 recovery observation (Roy et al. 2008). Here, only the last part a single burst was covered. From this detection and the fact that the average of Stokes V over a complete burst (almost completely) vanishes we infer that during an earlier part of the burst, Stokes V must have the opposite sign. In other words, if we can assume that the 2003 burst is similar to the 2002 bursts with regard to circularly polarised emission, there must be a sign change in the circular polarisation during the bursts.

\subsection{Maximum source size and maximum distance for incoherent emission}

All of the steep rising part of the bursts can be well approximated by a straight line. This is true even at the very beginning of the bursts, when the flux is at or just above the noise level. It can be seen in the lightcurve down to $10 \mathrm{~s}$ sampling, but at full $(5 \mathrm{~s})$ sampling we have insufficient signal to noise to trace any possible slope flattening down to the first $5 \mathrm{~s}$ of the beginning of the bursts. The average slope of the bursts in Table 3 is $0.75 \mathrm{Jy} / \mathrm{min}$ or $0.125 \mathrm{Jy} / 10 \mathrm{~s}$. This implies a flux doubling time of $\Delta t=10 \mathrm{~s}$ at the beginning of the bursts, when the flux is $125 \mathrm{mJy}$. The maximum source size at that time is then 10 lightseconds, if we assume that the source is not moving at a relativistic velocity (see, e.g., Harris et al. 2006, for some background). We can use the maximum source size $c \cdot \Delta t$ to link the brightness temperature $T_{\mathrm{b}}(\mathrm{K})$ to the flux $F$ and maximum distance $D$ (see, e.g., Rybicki \& Lightman 1979):

$T_{\mathrm{b}}=\frac{\lambda^{2} I_{v}}{2 k}=\frac{\lambda^{2} F}{2 k \pi \theta^{2}}=\frac{2 F}{\pi k}\left(\frac{D}{v \Delta t}\right)^{2}$

where $\lambda, I_{v}, v, k$ and $\theta$ are the wavelength, the specific intensity, the frequency, Boltzmann's constant and the angle subtended by the radius of the source, respectively. If we express the distance in pc, the flux in Jy and the frequency in $\mathrm{GHz}$, we get:

$T_{\mathrm{b}}=4.39 \times 10^{11} \mathrm{~F}\left(\frac{D}{v \Delta t}\right)^{2}$.

If synchrotron self-Compton radiation limits the brightness temperature to $10^{12} \mathrm{~K}$, the maximum distance for a source of size ten lightseconds and a flux of $0.125 \mathrm{Jy}$ emitting incoherently at $325 \mathrm{MHz}$ is $14 \mathrm{pc}$, assuming it is not moving at a relativistic velocity. Hyman et al. (2005) used the decay time of the bursts (conservatively estimated at $\simeq 2 \mathrm{~min}$ ) to calculate a maximum distance of $70 \mathrm{pc}$. So we have improved this upper limit by a factor 5 .

\section{Discussion}

Five of these upper limits on the flux of GCRT J1745-3009 come from the oldest observations of this field in the $92 \mathrm{~cm}$ band. This may provide interesting constraints on the feasibility of the double neutron star binary model (Turolla et al. 2005) in the near future. In this model, similar to J0737-3039, the period of recurrence of the 2002 bursts is explained by an orbital period of $77 \mathrm{~min}$. The lack of activity for many years is explained by geodetic precession, which could have caused the wind beam of the most luminous pulsar not to intercept the magnetosphere of the other pulsar for decades. Zhu \& Xu (2006) claim that the redetection in 2003 (Hyman et al. 2006) does not support this model. Their remark was, however, erroneously based on a geodetic precession period of $\simeq 3 \mathrm{yr}$, but this is actually $\simeq 21$ years $^{1}$. The last redetection (2004 March 20)

1 The "characteristic time for changing the system geometry" as mentioned by Turolla et al. (2005) differs from the period of geodetic precession by a factor $2 \pi$. 
and the first observation (1986 March 29) are 18 years apart. Unfortunately this timespan is too short to test the double neutron star binary model, but not if we redetect the system in the near future. More constraining are the results from population synthesis models (see, e.g., Portegies Zwart \& Spreeuw 1996, Fig. 2): fairly eccentric $(0.3<e<0.6)$ double neutron star binaries with an orbital period of 77 min are scarce, even compared to systems like J0737-3039. Also, the unpulsed emission needed for this model has not been detected in J0737-3039 (Chatterjee et al. 2005).

The lightcurve from our reduction of the 2002 discovery dataset shows that the bursts have similar shapes. There are three distinct parts separated by breaks, a steep rise, a gradual brightening and a steep decay. The main differences with the lightcurve from the discovery paper (Hyman et al. 2005) can probably be explained by sidelobes from SgrA (Roy et al. 2007, end of Sect. 2). These sidelobes are not seen in our images. Apparently, the lightcurve from the discovery paper was made by compiling fluxes from successive snapshot images (Hyman et al. 2006, paragraph 2). Therefore, we also made a lightcurve with $30 \mathrm{~s}$ sampling of the fourth burst using the AIPS task "IMAGR" and natural weighting, but the differences were negligible. We also learned that the output from "DFTPL" is likely to be more accurate than fluxes from snapshots (Greisen 2009, priv. comm.).

Our refined reduction of the discovery data seems to support the transient white dwarf model pulsar proposed by Zhang \& Gil (2005). A light-house beam associated with a highly magnetized white dwarf can emit radio emission with a 77 min period while maintaining an accuracy better than one second. The duty cycle $9 / 77 \simeq 0.1$ (with a few percent jitter from one pulse to another) is typical for pulsars. Moreover, an intensity asymmetry between the opposite sides of single pulses is typical in normal pulsars, so it can be expected also in white dwarf pulsars.

On the other hand, if the bursts we see are actually convolved with some scattering function, the intrinsic shape of the bursts could be different. Interstellar scattering can cause bursts to decay exponentially. We compared exponential fits to weighted linear regression for the $1 \mathrm{~min}$ data chunks that we used to determine the times of steepest decay. We found that residuals for linear fits are slightly smaller (12\% overall) than for exponential fits. The exponential fit was better than the linear fit for the tail of one of the four bursts only. From the exponential fits we found decay times of $0.56,0.77,0.73$ and $0.81 \mathrm{~min}$ for the second, third, fourth and fifth burst, respectively. These values are rather large for a source near the GC. For an observing frequency of $325 \mathrm{MHz}$ and for the position of GCRT J1745-3009 on the sky, pulse broadening times of 3.96-8.72 s and a DM of $567-751 \mathrm{~cm}^{-3} \mathrm{pc}$ are estimated from the NE2001 model of Cordes \& Lazio (2003), assuming a distance (to the GC) of $8 \mathrm{kpc}$ (Reid 1993). We also checked what dispersion measure would follow from our average scattering timescale $(0.72 \mathrm{~min})$ and the empirical relation found by Mitra \& Ramachandran (2001):

$\tau_{\mathrm{sc}}=4.5 \times 10^{-5} \cdot \mathrm{DM}^{1.6} \cdot\left(1+3.1 \times 10^{-5} \cdot \mathrm{DM}^{3}\right) \cdot \lambda^{4.4}$

with the scattering time $\left(\tau_{\mathrm{sc}}\right)$ in ms, the dispersion measure (DM) in $\mathrm{cm}^{-3} \mathrm{pc}$ and the observation wavelength $(\lambda)$ in meters. From this relation we find a dispersion measure of $\simeq 925 \mathrm{~cm}^{-3} \mathrm{pc}$. This would imply that GCRT J1745-3009 is located beyond the GC. For a check on consistency we compared this dispersion measure with the DM that can be found from the formula for the dispersion delay $\Delta t$ (in seconds):

$\Delta t=4150 \cdot \mathrm{DM} \cdot\left(\frac{1}{f_{1}^{2}}-\frac{1}{f_{2}^{2}}\right)$ between the highest $\left(f_{2}=327.50 \mathrm{MHz}\right)$ and lowest frequency IF ( $f_{1}=321.56 \mathrm{MHz}$ ) using the times of steepest rise for four of the bursts. The delay we found was $-0.94 \pm 3.65$ s corresponding to a DM of $-653 \pm 2530 \mathrm{~cm}^{-3} \mathrm{pc}$, consistent with the value above, but a very weak constraint.

From the poorer quality of the exponential fits relative to the linear fits we are inclined to conclude that the shape of the tails of the observed bursts are dominated by tails in the intrinsic emission. It seems justified that the average decay time from the exponential fits $(0.72 \mathrm{~min})$ is merely an upper limit for the true scattering time. In general we can state that for scattering times corresponding to distances not far beyond the Galactic Center the intrinsic burst shape will not differ greatly from the observed burst shape, besides any unresolved variability on very short timescales. The reason for this is that the duration of the observed bursts is much longer $(\simeq 10 \mathrm{~min})$ than any reasonable scattering time for sources near the GC.

We can work out the original burst profile using theorems for Laplace transforms. The intrinsic emission $I(t)$ is convolved with the scattering function $\zeta(t)$. This gives the observed burst $O(t)$ :

$O=I * \zeta$

where $*$ denotes convolution. For simple scattering, $\zeta$ is the product of the Heaviside step function $\Pi$ and an exponential:

$\zeta(t)=\Pi(t) \cdot \exp \left(\frac{-t}{\tau_{\mathrm{sc}}}\right)$.

The Laplace transform of this product is equal to $\frac{1}{s+\alpha}$, with $\mathrm{s}$ the transformed coordinate and $\alpha=1 / \tau_{\mathrm{sc}}$. Now, using the theorems for Laplace transforms of convolved functions and derivatives we find:

$I \cdot \kappa=\alpha \cdot O+\frac{\mathrm{d} O}{\mathrm{~d} t}$

with $\kappa$ a constant for normalization. If no emission is absorbed, it follows that $\kappa=\alpha$. Thus, we could reconstruct the intrinsic, unscattered burst from the observed burst if we knew the scattering time $\tau_{\mathrm{sc}}$. If the observed burst is represented very accurately by three straight lines for the steep rise, the gradual brightening and the steep decay, the original burst must have the same slopes. It then follows that $\tau_{\mathrm{sc}}=1 / \alpha=0$, hence no scattering, unless there are faults, i.e. sudden "jumps", in the intensity of the intrinsic emission. So the breaks link scattering times and fault sizes.

Without any assumptions on the possible degree of faulting in the intrinsic emission, we can find an upper limit for the scattering times using the end of the tails of the observed bursts. The slopes seem constant until the flux is essentially zero for at least three of the bursts. For the end of the tail of the second burst, which is relatively noisy, this is not so clear. Equation (7) then imposes an upper limit on the scattering time $\tau_{\mathrm{sc}}$ from the condition that the intrinsic emission cannot be negative. This means that the scattering time must be smaller than the time resolution for which we can determine the slopes with confidence: $10 \mathrm{~s}$. This implies that GCRT J1745-3009 cannot be located far beyond the GC. From the NE2001 model of Cordes \& Lazio (2003) we find a pulse broadening time of $9.93 \mathrm{~s}$ at $325 \mathrm{MHz}$ for a distance of $11 \mathrm{kpc}$ in the direction of GCRT J1745-3009.

We conclude from this discussion that the observed bursts depicted in Fig. 5 will closely resemble the intrinsic bursts. Models will need to explain the asymmetry of the bursts, the steep rise, the more gradual brightening and the steep decay and the breaks between them as well as the fact that the brightest emission is seen just before the steep decay. 


\section{Conclusions}

We have derived new upper limits on the quiescent emission of GCRT J1745-3009 at seven epochs. Six observations were made in the $92 \mathrm{~cm}$ band and one in the $21 \mathrm{~cm}$ band. The $92 \mathrm{~cm}$ observation of GCRT J1745-3009 on 2005 March 24 with the WSRT was the second deepest until that time. Five of these seven epochs constitute the oldest set of $92 \mathrm{~cm}$ observations taken of the Galactic Center. The nondetections at those epochs do not provide evidence for the double neutron star binary model (Turolla et al. 2005) with a geodetic precession period close to 18 years. However, geodetic precession times could well be somewhat longer.

We have reproduced the lightcurve of the discovery dataset of GCRT J1745-3009 more accurately and more completely than in the discovery paper. We see that the shapes of the five bursts are consistent: a steep rise, a gradual brightening and a steep decay. We have improved the $5 \sigma$ upper limit on interburst emission from $75 \mathrm{mJy}^{\text {beam }}{ }^{-1}$ to $31 \mathrm{mJy} \mathrm{beam}^{-1}$. Also, we further constrained the $5 \sigma$ upper limit on the fractional circular polarisation from $15 \%$ to $8 \%$. We determined the recurrence interval between bursts more accurately: $77.012 \pm 0.021 \mathrm{~min}$. We see no evidence for aperiodicity, but we do find that the duration of the bursts varies at the level of a few $\%$. We derived a very steep spectral index, $\alpha=-6.5 \pm 3.4$. We have investigated scattering and we have shown that scattering times must be less than $10 \mathrm{~s}$. This implies that GCRT J1745-3009 cannot be located far beyond the GC. It also means that the shape of the observed bursts will differ little from the intrinsic emission. Models for GCRT J1745-3009 have to explain the asymmetry in the shape of the bursts and in particular the gradual brightening until the steep decay. Some of the suggested models (Turolla et al. 2005; Zhu \& Xu 2006) predict symmetric bursts. The simplest interpretations of those models can now be ruled out, but it is conceivable that the asymmetry in the bursts could be achieved by adding some complexity to those models. Our results favour a rotating system, like the white dwarf pulsar (Zhang \& Gil 2005), because that can explain the high level of periodicity we see. We have shown that it is very unlikely that this transient is an incoherent synchrotron emitter, because it would have to be closer than $14 \mathrm{pc}$, unless the emitting region is moving at a relativistic velocity. Although we now have more contraints on the properties of this source, we are still unsure about its basic model.

A better understanding of its nature should come from more detections by long time monitoring with high sensitivity and high angular resolution, to tackle the confusion limit and to reduce the number of possible optical counterparts. The next generation of radio telescopes, like LOFAR (see, e.g., Fender et al. 2006), will help to do so. The most pressing issue in revealing the nature of GCRT J1745-3009 is still the determination of its distance, which could be achieved by a new detection with sufficient bandwidth between sidebands, in order to measure the time delay from dispersion towards the Galactic Center.

We have also investigated possible transient behaviour of a source on the opposite side of the supernova remnant G359.1-0.5 but we found no compelling evidence for variability.

Acknowledgements. We thank Tao An from Shanghai Astronomical Observatory for supplying us with an A-configuration model of the radio galaxy 1938-155. This model was necessary to phase calibrate our 1986 March 29 observations. We thank the anonymous referee for encouraging us to reproduce the lightcurve from the 2002 discovery dataset of GCRT J1745-3009 after we reported low noise levels in a map from that dataset. The National Radio Astronomy Observatory is a facility of the National Science Foundation operated under cooperative agreement by Associated Universities, Inc.

\section{References}

Baars, J. W. M., Genzel, R., Pauliny-Toth, I. I. K., \& Witzel, A. 1977, A\&A, 61, 99

Chatterjee, S., Goss, W. M., \& Brisken, W. F. 2005, ApJ, 634, L101

Condon, J. J., Cotton, W. D., Greisen, E. W., et al. 1998, AJ, 115, 1693

Cordes, J. M., \& Lazio, T. J. W. 2003 [arXiv:astro-ph/0301598], http://rsd-www.nrl.navy.mil/7213/lazio/ne_model/

Fender, R. P., et al. 2006, in Proceedings of the VI Microquasar Workshop, Microquasars and Beyond, 104

Greisen, E. W. 2003, in Information Handling in Astronomy - Historical Vistas, ed. A. Heck, Astrophysics and Space Science Library (Dordrecht, Netherlands: Kluwer Academic Publishers), 285, 109

Harris, D. E., Cheung, C. C., Biretta, J. A., et al. 2006, ApJ, 640, 211

Hyman, S. D., Lazio, T. J. W., Kassim, N. E., et al. 2005, Nature, 434, 50

Hyman, S. D., Lazio, T. J. W., Roy, S., et al. 2006, ApJ, 639, 348

Hyman, S. D., Roy, S., Pal, S., et al. 2007, ApJ, 660, L121

Kaplan, D. L., Hyman, S. D., Roy, S., et al. 2008, ApJ, accepted

Kulkarni, S. R., \& Phinney, E. S. 2005, Nature, 434, 28

Mitra, D., \& Ramachandran, R. 2001, A\&A, 370, 586

Nord, M. E., Lazio, T. J. W., Kassim, N. E., et al. 2004, AJ, 128, 1646

LaRosa, T. N., Kassim, N. E., Lazio, T. J. W., \& Hyman, S. D. 2000, AJ, 119, 207

Portegies Zwart, S. F., \& Spreeuw, H. N. 1996, A\&A, 312, 670

Reid, M. J. 1993, ARA\&A, 31, 345

Roy, S., Hyman, S. D., Pal, S., et al. 2007, in Proceedings of Bursts, Pulses and Flickering: wide-field monitoring of the dynamic radio sky, 12-15 June, Kerastari, Tripolis, Greece, 9

Roy, S., Hyman, S. D., Pal, S., et al. 2008, in Proc. 25th meeting of ASI (2007), 25

Rybicki, G. B., \& Lightman, A. P. 1979, in Radiative Processes in Astrophysics (New York, NY: Wiley), 8, 25

Turolla, R., Possenti, A., \& Treves, A. 2005, ApJ, 628, L49

Zhang, B., \& Gil, J. 2005, ApJ, 631, L143

Zhu, W. W., \& Xu, R. X. 2006, MNRAS, 365, L16 